\title{
Association between cigarette smoking and colorectal cancer sidedness: A multi-center big-data platform-based analysis
}

\author{
Lu-Ping Yang ${ }^{1 \dagger}$, Zi-Xian Wang ${ }^{1 \dagger}$, Rui Zhang ${ }^{2}$, Na Zhou ${ }^{3}$, A-Man Wang ${ }^{4}$, Wei Liang ${ }^{6}$, Zhi-Qiang Wang ${ }^{1}$, \\ Hui-Yan Luo ${ }^{1}$, Feng Wang ${ }^{1}$, Ji-Wei Liu ${ }^{4}$, Fang Liu ${ }^{2}$, Xiao-Chun Zhang ${ }^{3^{*}}$, Yun-Peng Liu ${ }^{5^{*}}$ and Ying Jin ${ }^{1 *}$
}

\begin{abstract}
Background: Sidedness (right/left) of colorectal cancer (CRC) is essential for treatment. Whether carcinogenesis of tobacco varies by sidedness remains unclear. The present study aims to evaluate the sidedness tendency of cigarette smoking and to explore its impact on prognosis.

Methods: In the multi-center retrospective study, data on 46166 Chinese CRC patients were extracted from a big-data platform. Logistic regression analyses were performed to evaluate qualitative and quantitative associations between smoking and tumor sidedness. Survival analyses were conducted in metastatic CRC.

Results: History of smoking was associated with left-sided CRC (LSCRC; Adjusted odds ratio, 1.25; 95\% Cl, $1.16-1.34$; $P<.001)$. The sidedness tendency towards LSCRC increased from non-smokers, to ex-smokers, and to current smokers ( $P$ for trend $<.001)$. Longer duration $(P$ for trend $<.001)$ and larger total amount of cigarette smoking $(P$ for trend $<.001)$ were more associated with $L S C R C$, respectively. The association was confirmed in both left-sided colon cancer and rectal cancer, but was stronger for rectal cancer $(P=.016)$. Alcoholism significantly enhanced the association by $7 \%$ $(P=$.027). Furthermore, prognostic advantage of metastatic LSCRC diminished among ever-smokers, with contrary survival impacts of smoking on either side of CRC.

Conclusions: History of smoking was associated with LSCRC in a positive dose-response relationship, and presented opposite prognostic impacts on right- and left-sided tumors. Smoking potentially plays an instrumental role in the mechanism for sidedness heterogeneity in CRC.
\end{abstract}

Keywords: Cigarette smoking, Colorectal cancer, Sidedness, Big-data platform, Prognosis

*Correspondence: zxc9670@qdu.edu.cn; ypliu@cmu.edu.cn; jinying1@sysucc. org.cn

${ }^{\dagger}$ Lu-Ping Yang and Zi-Xian Wang contributed equally to this work ${ }^{1}$ State Key Laboratory of Oncology in South China, Collaborative Innovation Center for Cancer Medicine, Sun Yat-Sen University Cancer Center, 651 Dong Feng Road East, Guangzhou 510060, China

${ }^{3}$ Precision Medicine Center of Oncology, The Affiliated Hospital of Qingdao University, No. 59 Haier Road, Qingdao 266016, China

${ }^{5}$ Department of Medical Oncology, The First Hospital of China Medical University, Shenyang 110001, China

Full list of author information is available at the end of the article

\section{Introduction}

Colorectal cancer (CRC) is the third most common cancer and its mortality ranks fourth worldwide $[1,2]$. In China, the increasingly high incidence of CRC has roused intense attention, and the identification of etiologic and prognostic factors is an important task for oncologists.

Modifiable behaviors, including smoking, chronic alcohol intake, lack of physical exercise, and unhealthy diet, are risk factors for CRC [3, 4]. Previous studies have demonstrated a significant association of cigarette smoking with CRC incidence and mortality [5, 6]. Researchers

c) The Author(s) 2021. This article is licensed under a Creative Commons Attribution 4.0 International License, which permits use, sharing, adaptation, distribution and reproduction in any medium or format, as long as you give appropriate credit to the original author(s) and the source, provide a link to the Creative Commons licence, and indicate if changes were made. The images or other third party material in this article are included in the article's Creative Commons licence, unless indicated otherwise in a credit line to the material. If material is not included in the article's Creative Commons licence and your intended use is not permitted by statutory regulation or exceeds the permitted use, you will need to obtain permission directly from the copyright holder. To view a copy of this licence, visit http://creativeco mmons.org/licenses/by/4.0/. The Creative Commons Public Domain Dedication waiver (http://creativecommons.org/publicdomain/ zero/1.0/) applies to the data made available in this article, unless otherwise stated in a credit line to the data. 
have evaluated the association in subgroups of different primary tumor locations, and some proved it more solid for rectal cancer (RC) rather than colon cancer. This finding implied a differential susceptibility of carcinogenesis by cigarettes between subsites of colorectum. However, inconsistent conclusions were obtained from previous studies and none of them conducted a direct comparison between subsites. [6-10].

In recent years, the theory that right-sided colon cancer (RSCC) and left-sided colorectal cancer (LSCRC) are biologically heterogeneous has been established and widely accepted, but the underlying mechanism has not been clear yet [11]. We wondered whether smokingrelated cancer susceptibility might vary between RSCC and LSCRC, and consequently result in the difference of tumor sidedness tendency between ever and never smokers and in the heterogeneity of molecular features between sidedness. It might also play a part in the prognostic advantage of LSCRC over RSCC among metastatic CRC (mCRC) [12, 13].

In this retrospective study, we evaluated the relationship between cigarette smoking and CRC sidedness based on a multi-center big-data intelligence platform with the aim of exploring potential tendencies for sidedness related to cigarette smoking, and assessed its potential prognostic influence regarding tumor sidedness.

\section{Methods}

\section{Data source and study population}

All data used in the study were extracted from the Bigdata Alliance for Colorectal Cancer (BACC) platform (YiduCloud Technology Ltd., Beijing, China) which is a big-data intelligence platform that integrates and converges mass multi-source heterogeneous electronic health-records data from multiple medical centers or hospitals all over China.

A total of 71429 Chinese CRC patients from the BACC platform diagnosed from January 1, 2000 to April 30, 2018 comprised the study population. The inclusion criteria were as follows: (i) the patient was diagnosed with colorectal adenocarcinoma by histopathology and (ii) was $\geq 18$ years old. The exclusion criteria were as follows: (i) information for confirming CRC sidedness was unavailable; (ii) information about the patient's history of smoking, alcohol or familial malignancy was unavailable. Additional file 1: Figure S1 shows the flowchart of the patient selection process. The final cohort for the analyses consisted of 46166 patients.

The study was approved by the Institute Research Ethics Committee at the Sun Yat-sen University Cancer Center (ID: B2018-154-01), and was conducted in accordance with best clinical practices and the Declaration of
Helsinki. All authors had access to the study data and reviewed and approved the final manuscript.

\section{Definitions of the study variables}

History of smoking, alcohol intake, and familial malignancy were extracted from the electronic medical records and structured in the BACC platform. Patients who reported a history of smoking were identified as eversmokers; those who had quit smoking for $\geq 3$ months were classified as ex-smokers and the rest ever-smokers were identified as current smokers. The quantification of cigarette smoking was measured in terms of daily consumption (i.e., the number of cigarettes smoked per day), duration (years) of smoking, and a smoking index which is the product of the former two measurements (cigarettes * years). The cut-off points for the number of cigarettes smoked per day and the duration (years) of smoking were adopted from a previous study [14], and the median value of the smoking index was used as its cut-off point.

The primary tumor location was defined in accordance to the diagnosis recorded in the BACC platform, and was used to clarify the definition of sidedness. RSCC was defined as a tumor in the cecum, ascending colon, or transverse colon. LSCRC was defined as a tumor located from the splenic flexure to the rectum. Left-sided colon cancer (LSCC) was defined as LSCRC excluding RC.

\section{Statistical analysis}

Categorical variables were analyzed using the Chi-square test. Temporal trends in the prevalence of smoking were evaluated using the Cochran-Armitage trend test. The associations between cigarette smoking and CRC sidedness were examined using binary logistic regression. In the logistic regression, we labelled RSCC as the reference. The odds ratios (OR) and 95\% CIs were estimated after adjustments for age at diagnosis, sex, and family history of malignancy, which have been reported to be potential confounders[15]. Multicollinearity of the regression models was determined by variance inflation factor (VIF); a VIF $\leq 10$ indicates a robust model with insignificant influence by collinearity. Given the stronger carcinogenic impact of smoking on the rectum, [5] we stratified LSCRC to LSCC and RC in the correlation analyses. We conducted subgroup analyses in subsets of patients by age $(18-39 / 40-59 / 60-79 / \geq 80$ years), sex (males/ females), year of diagnosis $(2000-2011 / 2012-2014 / 2$ $015-2018$ ), family history (yes/no), history of chronic alcohol intake (yes/no), and metastatic status at initial diagnosis (M1/M0). The ORs were compared in binary subgroups on the log scale. [16].

We further explored the prognostic effects of smoking and tumor sidedness on mCRC. The survival 
analyses were restricted to the patients with mCRC whose follow-ups lasted 3 months or longer (cohort $\mathrm{M}, \mathrm{n}=2$ 272), using Kaplan-Meier method with logrank test for comparisons of overall survival (OS). Multivariate Cox analyses including variables with $P$-values $<0.05$ in univariate Cox analyses were also conducted.

All statistical analyses were performed using SPSS Statistics Version 22.0 (IBM Corp, Armonk, NY), R Version 3.5.3 (The R Foundation, Vienna, Austria), and GraphPad Prism Version Version 7.04 (GraphPad Software, San Diego, CA). A two-tailed $P$-value $<0.05$ was considered statistically significant.

\section{Results}

Characteristics of the study population

Demographic and clinical characteristics of the study cohort are shown in Table 1. Among the 46166 eligible patients, the median age at diagnosis was 61 years (range, $18-102)$ and $60.4 \%$ were males. Nearly half $(46.3 \%)$ of the cases were diagnosed in the past three years. The majority $(63.3 \%)$ of the tumors were located in the rectum, followed by $22.8 \%$ with LSCC and $13.8 \%$ with RSCC.

This study consisted of 10712 (23.2\%) ever-smokers and $35454(76.8 \%)$ non-smokers. They were diagnosed at a similar age $(P=0.140)$, but a noticeable gender imbalance was found among the ever-smokers of whom $95.2 \%$ were males. More than half of the smokers exhibited concurrent alcoholism. A statistically significant increase

Table 1 Demographic and clinical characteristics of the study population and cohorts according to cigarette and alcohol intake status

\begin{tabular}{|c|c|c|c|c|}
\hline \multirow[t]{2}{*}{ Variable } & \multirow[t]{2}{*}{ All cases $(N=46,166)$} & \multicolumn{3}{|c|}{ Cigarette smoking status } \\
\hline & & $\operatorname{Ever}(n=10,712)$ & $\operatorname{Never}(n=35,454)$ & $P$ value \\
\hline Age, years & & & & $.14^{\mathrm{a}}$ \\
\hline $18-39$ & $2359(5.11 \%)$ & $371(3.46 \%)$ & $1988(5.61 \%)$ & \\
\hline $40-59$ & $18,028(39.05 \%)$ & $4438(41.43 \%)$ & $13,590(38.33 \%)$ & \\
\hline $60-79$ & $23,297(50.46 \%)$ & $5504(51.38 \%)$ & $17,793(50.19 \%)$ & \\
\hline$\geq 80$ & $2482(5.38 \%)$ & 399 (3.72\%) & $2083(5.88 \%)$ & \\
\hline Sex & & & & $<.001$ \\
\hline Male & $27,869(60.37 \%)$ & $10,202(95.24 \%)$ & $17,667(49.83 \%)$ & \\
\hline Female & $18,297(39.63 \%)$ & $510(4.76 \%)$ & $17,787(50.17 \%)$ & \\
\hline Year of diagnosis & & & & $<.001$ \\
\hline $2000-2011$ & $5962(12.91 \%)$ & $1131(10.56 \%)$ & $4831(13.63 \%)$ & \\
\hline $2012-2014$ & $18,822(40.77 \%)$ & $4274(39.90 \%)$ & $14,548(41.03 \%)$ & \\
\hline $2015-2018$ & $21,382(46.32 \%)$ & 5307 (49.54\%) & $16,075(45.34 \%)$ & \\
\hline Primary tumor location & & & & $<.001$ \\
\hline $\begin{array}{l}\text { Cecum, ascending and transverse } \\
\text { colon cancer }\end{array}$ & $6393(13.85 \%)$ & 1175 (10.97\%) & $5218(14.72 \%)$ & \\
\hline $\begin{array}{l}\text { Splenic flexure, descending and } \\
\text { sigmoid colon cancer }\end{array}$ & $10,548(22.85 \%)$ & $2378(22.20 \%)$ & $8170(23.04 \%)$ & \\
\hline Rectum & $29,225(63.30 \%)$ & $7159(66.83 \%)$ & $22,066(62.24 \%)$ & \\
\hline Primary tumor sidedness & & & & $<.001$ \\
\hline Right-sided & $6393(13.85 \%)$ & 1175 (10.97\%) & $5218(14.72 \%)$ & \\
\hline Left-sided & $39,773(86.15 \%)$ & 9537 (89.03\%) & $30,236(85.28 \%)$ & \\
\hline Alcohol intake history & & & & $<.001$ \\
\hline No & $37,819(81.92 \%)$ & 4701 (43.89\%) & $33,118(93.41 \%)$ & \\
\hline Yes & 8347 (18.08\%) & $6011(56.11 \%)$ & 2336 (6.59\%) & \\
\hline Metastasis at diagnosis & & & & $<.001$ \\
\hline No & $15,523(33.62 \%)$ & $3440(32.11 \%)$ & $12,083(34.08 \%)$ & \\
\hline Yes & 8567 (18.56\%) & $1889(17.63 \%)$ & $6678(18.84 \%)$ & \\
\hline Unknown & 22,076 (47.82\%) & $5383(50.25 \%)$ & $16,693(47.08 \%)$ & \\
\hline Family history & & & & $<.001$ \\
\hline Yes & 4264 (9.24\%) & $1256(11.73 \%)$ & 3008 (8.48\%) & \\
\hline No & 41,902 (90.76\%) & $9456(88.27 \%)$ & 32,446 (91.52\%) & \\
\hline
\end{tabular}

\footnotetext{
a $P$ value of student's t test to compare means
} 
was found among patients with CRC who smoked (from $19.0 \%$ in $2000-2011$ to $24.8 \%$ in $2015-2018$ ) in both genders (Fig. 1a). Females showed a more pronounced increase in the magnitude of smokers (by 66.7\%). The smoking trends implied that except bans on public indoor smoking, cigarette smoking remained a crucial public health issue. In terms of clinical features of patients with different tobacco-consuming amount, we observed that the proportion of elderly, male patients or patients with LSCRC, history of chronic alcoholic intake or family history of cancer tended to grow as the increment of cigarettes smoked (Fig. 1b).

\section{Sidedness tendency}

Table 2 shows the odds ratios of the sidedness tendency towards LSCRC over RSCC by smoking status. A history of smoking was found to be associated with LSCRC (Adjusted odds ratio [AOR], 1.25; 95\% CI, $1.16-1.34 ; P<0.001)$. Patients who quit smoking showed no tendency towards either side, and current smokers showed a significant tendency towards LSCRC ( $P$ for trend $<0.001)$. Moreover, a stronger dose-response relationship was found for a longer duration of smoking $(P$ for trend $<0.001)$ and a larger smoking index $(P$ for trend $<0.001$ ) with LSCRC. Among heavy smokers with a smoking history $>30$ years or a smoking index $>600$, the likelihood to develop LSCRC other than RSCC was respectively, $55 \%$ and $46 \%$ higher than for non-smokers. However, a higher number of cigarettes smoked per day did not indicate a stronger tendency towards LSCRC. These findings present a trend in the long-term effect of cigarette smoking on CRC sidedness. No multicollinearity was observed to confound the association (See Additional file 2 Table S1). We further adjusted for the geographic location of medical centers and observed consistent results (See Additional file 2 Table S2).

The multivariate analysis showed that females tended to develop RSCC (AOR, 0.78; 95\% CI, $0.73-0.83 ; P<0.001$ ), as did patients with a family history of malignant tumors (AOR, 0.64; 95\% CI, 0.59-0.69; $P<0.001$ ). Furthermore, there was no significant association between a history of alcohol consumption and sidedness of CRC (AOR, 1.06; 95\% CI, $0.96-1.15 ; P=0.110$ ). However, the sidedness tendency of cigarette smoking tended to grow stronger from never smokers, non-drinker smokers, to drinkersmokers (AOR for non-drinker smokers, 1.21; 95\% CI, 1.09-1.33; AOR for drinker-smokers, 1.28; $95 \% \mathrm{CI}$, $1.17-1.41 ; P$ for trend $<0.001$ ). Additional file 2: Table S3 presents the distribution of clinical variables across the tumor sidedness.

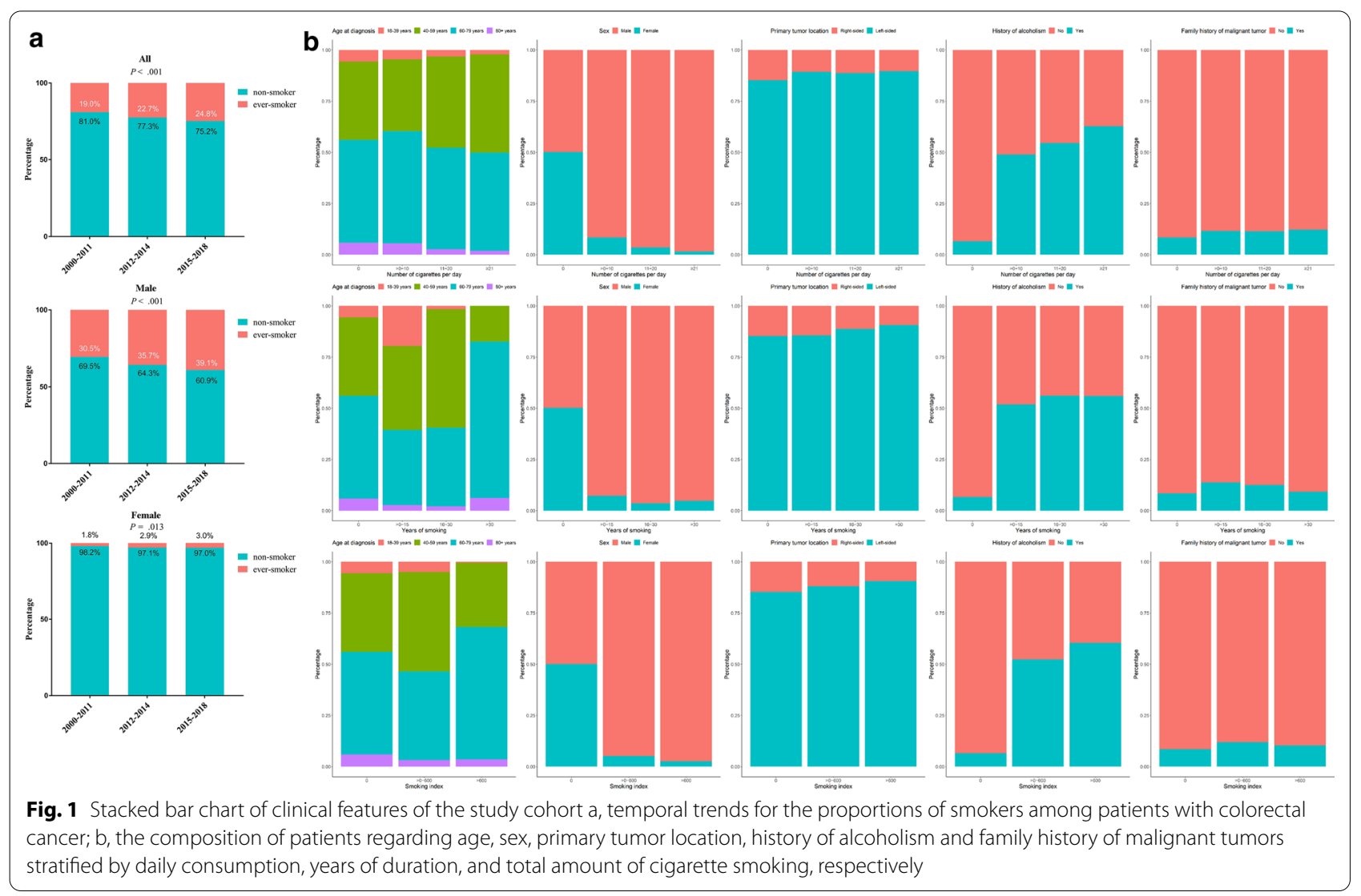


Table 2 Adjusted odds ratios for sidedness of colorectal cancer (left-sided vs. right-sided) according to smoking history

\begin{tabular}{|c|c|c|c|}
\hline & No. of cases & $\operatorname{AOR}(95 \% \mathrm{Cl})^{\mathrm{a}}$ & $P$ for trend ${ }^{b}$ \\
\hline \multicolumn{4}{|l|}{ Cigarette smoking } \\
\hline Never & $30,236 / 5218$ & Reference & \multirow[t]{2}{*}{$<.001$} \\
\hline Ever & $9537 / 1175$ & $1.25(1.16,1.34)$ & \\
\hline \multicolumn{4}{|c|}{ Current smoking status } \\
\hline Non-smokers & $30,236 / 5218$ & Reference & \multirow[t]{4}{*}{$<.001$} \\
\hline Ex-smokers & $2570 / 287$ & $0.90(0.80,1.02)$ & \\
\hline Current smokers & $2215 / 388$ & $1.39(1.22,1.58)$ & \\
\hline Unknown cessation & $4752 / 500$ & $1.43(1.29,1.58)$ & \\
\hline \multicolumn{4}{|c|}{ Number of cigarettes per day } \\
\hline 0 & $30,236 / 5218$ & Reference & \multirow[t]{5}{*}{.790} \\
\hline$>0-10$ & $2178 / 259$ & $1.32(1.16,1.52)$ & \\
\hline $11-20$ & $4382 / 557$ & $1.19(1.08,1.32)$ & \\
\hline$\geq 21$ & $1568 / 181$ & $1.30(1.11,1.53)$ & \\
\hline Unknown & $1409 / 178$ & $1.24(1.06,1.46)$ & \\
\hline \multicolumn{4}{|c|}{ Duration of smoking (years) } \\
\hline 0 & $30,236 / 5218$ & Reference & \multirow[t]{5}{*}{$<.001$} \\
\hline$>0-15$ & $1025 / 173$ & $0.98(0.83,1.16)$ & \\
\hline $16-30$ & $4349 / 551$ & $1.18(1.07,1.30)$ & \\
\hline$>30$ & $3016 / 310$ & $1.55(1.36,1.76)$ & \\
\hline Unknown & $1147 / 141$ & $1.27(1.06,1.52)$ & \\
\hline \multicolumn{4}{|l|}{ Smoking index } \\
\hline 0 & $30,236 / 5218$ & Reference & \multirow[t]{4}{*}{$<.001$} \\
\hline$>0-600$ & $4883 / 657$ & $1.14(1.04,1.25)$ & \\
\hline$>600$ & $2777 / 289$ & $1.46(1.28,1.66)$ & \\
\hline Unknown & $1877 / 229$ & $1.29(1.12,1.49)$ & \\
\hline
\end{tabular}

a After adjustment for age, sex, and family history

${ }^{b} P$ value was evaluated based on the cases without unknown data

\section{Sidedness tendency stratified by intestinal segment}

Among the patients diagnosed with LSCRC, the primary tumor for 10548 (26.5\%) was located in the colon, and for $29225(73.5 \%)$ it was located in the rectum. Smokers tended to have LSCC rather than RSCC (AOR, 1.13; 95\% CI, $1.04-1.23 ; P=0.010)$, and to have $\mathrm{RC}$ rather than RSCC (AOR, 1.30; 95\% CI, 1.20-1.40; $P<0.001$ ). Both intestinal segments showed solid associations with a history of smoking, while a significantly stronger tendency towards sidedness was found in $\mathrm{RC}(P=0.016)$. Similar results were obtained in the dose-response analyses (Fig. 2). As the duration and total amount of cigarette consumption increased, gaps between the associating strengths of LSCC and RC became wider, indicating that the sidedness tendency towards RC was more sensitive to long-term dose accumulation of tobacco compared with that towards LSCC. Smoking cessation did not significantly reverse the trend in sidedness for the patients with colon cancer; however, ex-smokers were more likely to develop RSCC than RC (AOR, 0.84; 95\% CI, $0.75-0.95$ ).

\section{Subgroup analyses of sidedness tendencies}

Chronic alcohol intake, which is usually concurrent with a habit of smoking, significantly enhanced the sidedness tendency towards LSCRC $(P=0.027)$. Among the patients diagnosed at $\geq 40$ years old, a significant association was found between cigarette smoking and LSCRC, which tended to increase with age; however, this tendency was attenuated among the younger patients (aged 18 to 39 years), possibly due to inadequate accumulation of cigarettes. The sidedness tendency was robust in the subgroups classified by sex, history of alcohol intake, or family history of malignant cancer. Figure 3 shows the AORs for LSCRC compared to RSCC in these subgroups.

\section{Prognostic impact of sidedness influenced by smoking status}

Since OS differed between metastatic LSCRC and RSCC, the prognostic impact of cigarette smoking on heterogeneity of sidedness in mCRC was in need of clarification. The median follow-up duration of cohort $M(n=2272)$ was 31.12 months, and the clinical features are shown in Additional file 2: Table S4. Cigarette smoking was not proven to be an independently prognostic factor (Hazard ratio $[\mathrm{HR}], 1.00 ; 95 \% \mathrm{CI}, 0.87-1.14 ; P=0.961$; See Fig. 4a). The prognosis of patients with LSCRC was significantly more favorable ( $\mathrm{HR}, 0.85$; $95 \% \mathrm{CI}, 0.74-0.97$; $P=0.017$; See Fig. 4b), which was consistent with previous researches. Similar results were obtained in nonsmokers in that they had a better prognosis for LSCRC over RSCC (HR, 0.79; 95\% CI, 0.67-0.92; $P=0.002$; See Fig. 4c), while the prognostic advantage of LSCRC was eliminated among the ever-smokers (HR, 1.09; 95\% CI, $0.80-1.49 ; P=0.597$; See Fig. $4 d$ ). An interesting result was observed and might be the underlying reason, that is, a history of smoking relatively elongated the median OS of patients with RSCC by 2.7 months and on the contrary shortened that of patients with LSCRC by 6.1 months (although not statistically significant; See Fig. 4e and Additional file 2: Table S5). For patients with LSCRC, heavy smokers with smoking index $>600$ showed marginally poorer prognosis ( $\mathrm{HR}, 1.22$; 95\% CI, $0.99-1.49$; $P=0.059)$; however, numerically lower HR was observed in RSCC (See Additional file 2: Table S6). It implied that cigarette smoking was potentially a favorable prognostic factor for RSCC and an unfavorable factor for LSCRC. Furthermore, we observed an insignificant reduce of OS by smoking in patients with metastatic LSCRC who received cetuximab (See Fig. $4 \mathrm{f}$ ). It could not explain the loss of prognostic advantage for metastatic LSCRC and further investigation was needed. 


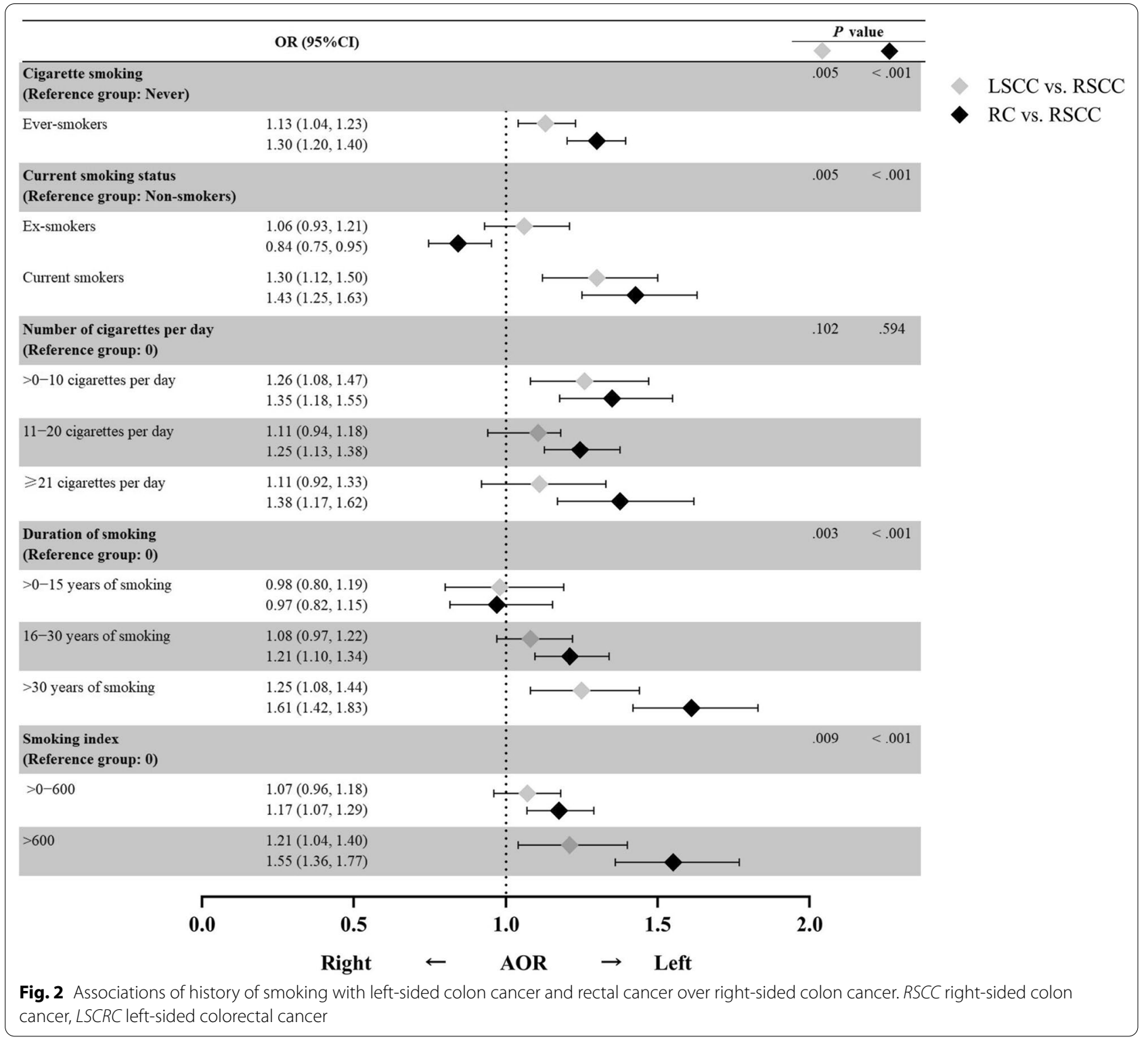

\section{Discussion}

In recent years, concerns have been raised that sidedness of colorectal cancer could influence biological behaviors of the tumor $[11,12,17]$. The difference in etiology might be the underlying mechanism of the heterogeneity between RSCC and LSCRC. Cigarette smoking, as a risk factor for mortality of CRC introducing genetic alterations and epigenetic imprints [18, 19], has the possibility to attribute to the heterogeneity. Thus, we evaluated the hypothesis in the present study based on 46166 Chinese patients from the multi-center BACC platform, and proved cigarette smoking to have a positive doseresponse relationship with LSCRC. A 2006 study by Zissman et al. reported an association between tobacco use and distal CRC [8], which was consistent with the results of our study; however, it lacked the analysis of proximal CRC, and the information has not been updated in recent years and was not confirmed in Asian populations. Liang et al. found a higher risk of developing cancer in the rectum than the colon among smokers, [6] but there were three studies showed inconsistent findings $[7,9,10]$. These results were controversial, and also raised the concern that the sidedness tendency towards LSCRC was, by nature, towards RC, not LSCC. The inconsistent results in terms of colon cancer subsites might be attributed to the different sample sizes and analytic methods. Those previous studies conducted indirect comparisons of CRC risks including healthy populations. In the present study, 


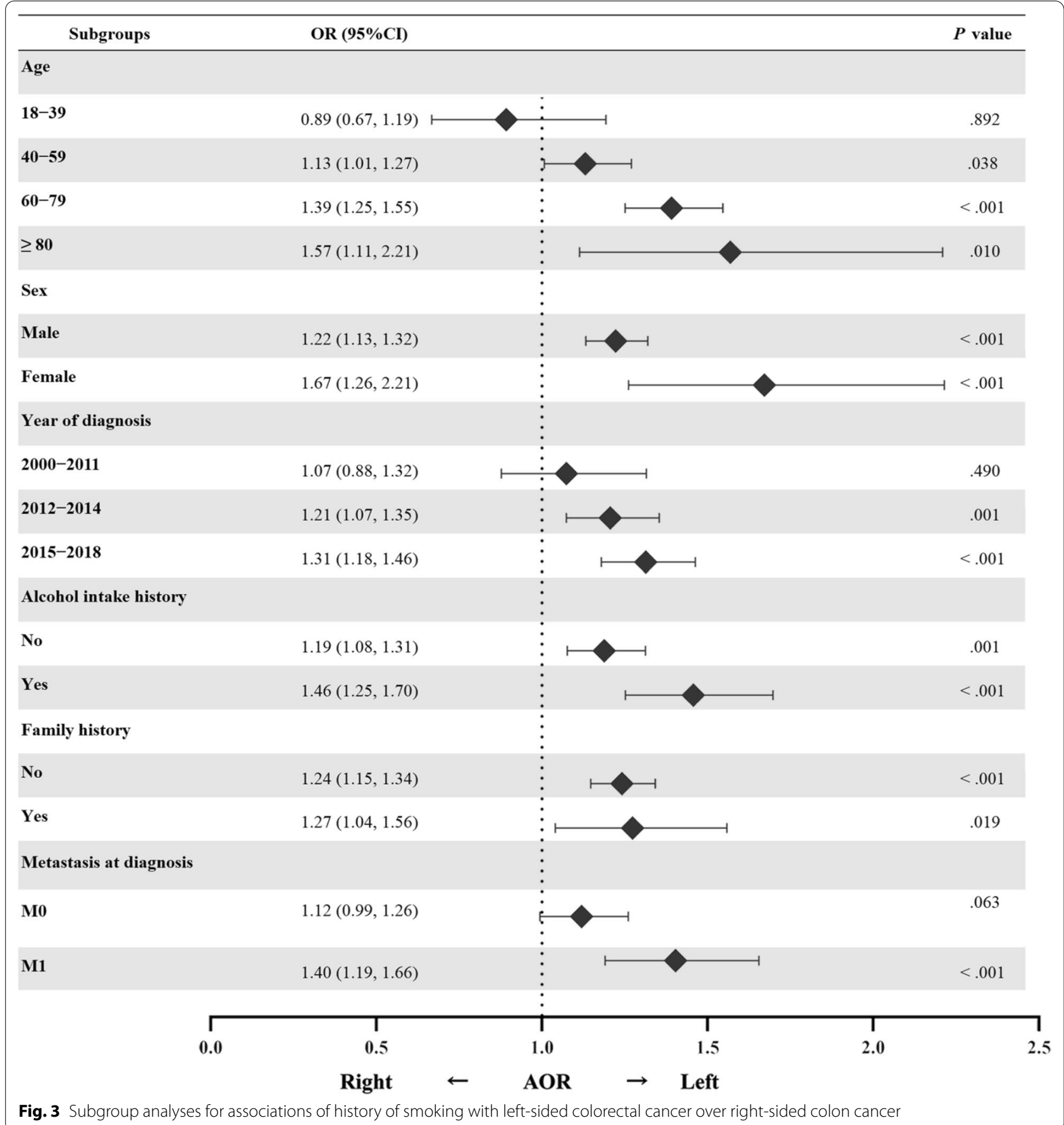

Fig. 3 Subgroup analyses for associations of history of smoking with left-sided colorectal cancer over right-sided colon cancer

(See figure on next page.)

Fig. 4 Survival curves of patients with metastatic colorectal cancer stratified by tumor sidedness and history of smoking. a-b, survival curves of cohort M stratified by history of smoking (a) or by tumor sidedness (b); c-d, survival curves of never smokers (c) or ever-smokers (d) stratified by tumor sidedness; $\mathbf{e}$, comparisons of overall survival regarding tumor sidedness and history of smoking; $\mathbf{f}$, survival curves of patients with metastatic LSCRC treated with cetuximab stratified by history of smoking. $m C R C$ metastatic colorectal cancer, RSCC right-sided colon cancer, LSCRC left-sided colorectal cancer. ${ }^{* * *}$ stands for $<.001$; ns stands for not significant. CRC colorectal cancer 


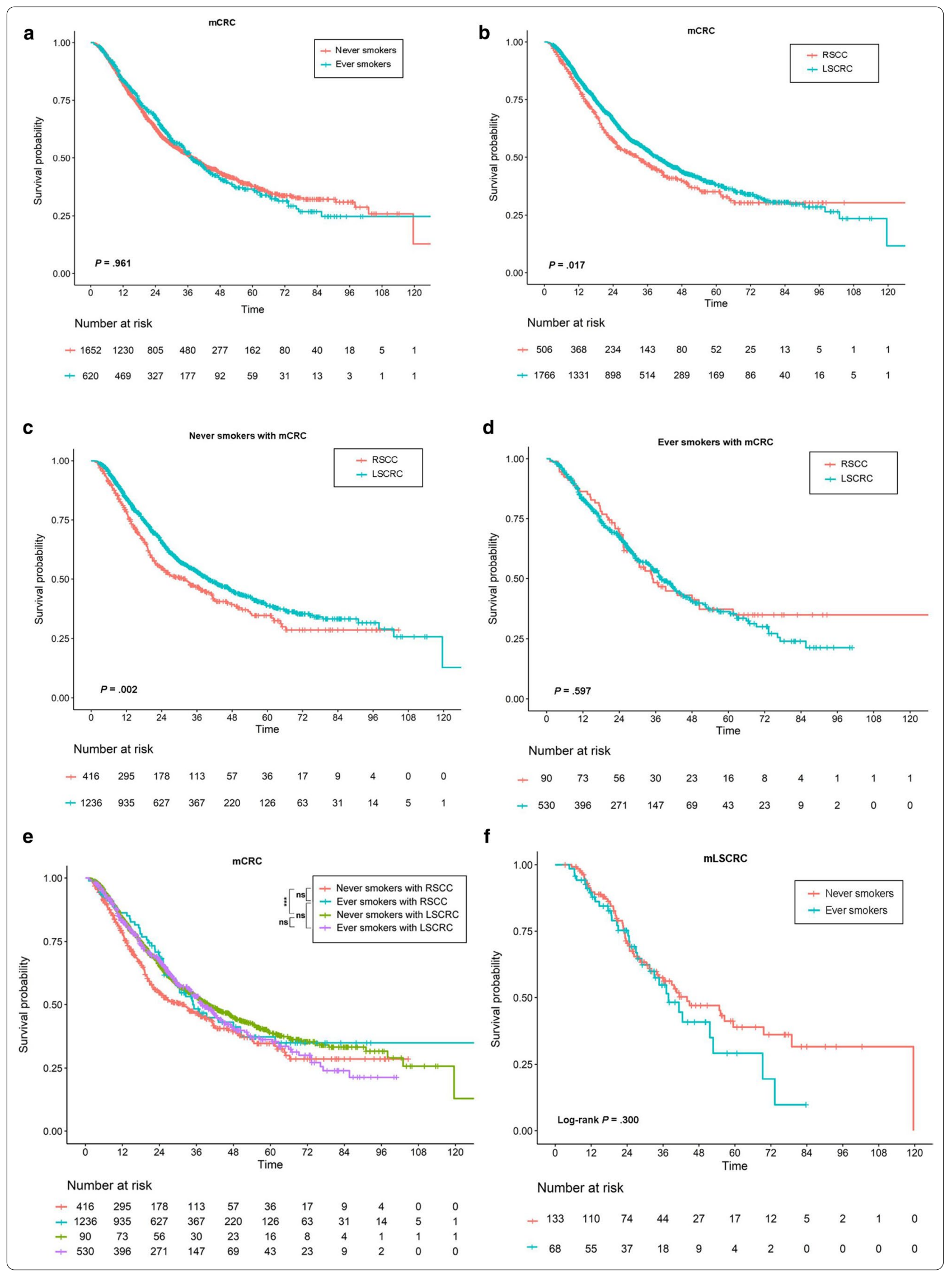


we conducted a direct comparison among patients in a large population from multiple medical centers; the doserelationship analyses improved the credibility of our findings. Moreover, we stratified LSCRC to LSCC and RC and validated the association respectively.

Hecht reported the presence of sixty carcinogens in cigarette smoke and more than sixteen in unburned tobacco, among which tobacco-specific nitrosamines, polycyclic aromatic hydrocarbons and aromatic amines possibly play a key role in carcinogenesis [18]. Analyses of corresponding genetic alterations have found cigarette smoking was in relation to MSI-H, CIMP-H, BRAF mutation, p53 mutation, APC mutation, and promoter 1A methylation in CRC [19-21]. The association of CRC sidedness with cigarette smoking which we observed in this study supported the hypothesis that tobacco has more intensive carcinogenic effects on LSCRC, and consequently, leave specific tobacco-related molecular signatures in LSCRC different from RSCC. LSCRC has been found to be associated with CIN, p53 mutation and NRAS mutation over RSCC [22-24]. Therefore, p53 mutation might be the molecular feature left by tobacco tending to LSCRC. Further bioinformatic studies are needed to uncover specific mechanisms behind tumorigenesis.

The impact of cigarette smoking on prognosis or response to therapy of CRC was not clear yet. We explored it among patients with $\mathrm{mCRC}$ since left-sided tumor location has been proved a favorable prognostic indicator $[17,25]$. However, in the present study, a history of smoking was neither a positive nor a negative prognostic factor of $\mathrm{mCRC}$, and it even relatively prolonged OS among patients with metastatic RSCC and diminished the prognostic advantage of LSCRC, which was a novel discovery and had not been reported before. Meanwhile, we observed numerically-shortened OS in smokers with LSCRC and thus speculated smoking might reduce the efficacy of the targeted drug specially for LSCRC, cetuximab, for instance. We found comparable efficacy of cetuximab between ever-smokers and never-smokers with LSCRC; previous studies suggested that smoking habit might suppress survival benefit from second-line cetuximab-based treatment, though [26]. In other types of cancer, there was evidence that cigarette smoking was not unfavorable for prognosis as presumed [27-30]. Smoking could even protect patients with apolipoprotein $\mathrm{E}$ positive breast cancer from anticancer-treatment-induced cognitive impairment. Several researches reported that smoking had potentials to improve efficacy of immunotherapy among patients with non-small-cell lung cancer, probably by introducing neoantigens, increasing tumor mutation burden, or upregulating PD-L1 expression. $[31,32]$. All these findings implied that despite carcinogenesis by tobacco, a history of smoking did not necessarily indicate a worse prognosis or less efficacy of treatment. This might lighten the mental burden of cancer patients who attributed smoking to the mortality and suffered from self-accusation. Nevertheless, smoking cessation was strongly recommended [32]. On the other hand, the different influences of smoking on survival between sidedness (See Additional file 2: Table S6) also suggested that tobacco alongwith its products might have heterogeneous biological effects in right- and left-sided tumors.

To the best of our knowledge, this is the first study to investigate the association between cigarette smoking and CRC sidedness based on a large population. On one hand, these findings suggest novel epidemiological features of CRC and its sidedness; on the other hand, new evidence is provided that biological heterogeneity could be partially caused by imbalanced carcinogenic effects of smoking between RSCC and LSCRC.

The present study has several limitations. First, information about cigarette -smoking history was collected through enquiry during the patients' first admission, and might have recall bias and inaccuracies, especially for numerical information. To diminish these effects, we converted continuous variables into categorical ones. Second, incomplete follow-up information limited larger scale survival analyses. Updated follow-ups and cohort expansion are needed for further validation. Third, molecular features were not included in the analyses due to limited data available; studies should investigate possible molecular mechanisms in the future.

In conclusion, among patients with CRC, a history of cigarette smoking was associated with LSCRC in a positive dose-response relationship, and was largely dependent on long-term accumulation. Smoking cessation weakened the association while history of concurrent alcoholism could enhance it. Smoking was not unfavorable for prognosis and it showed varied tendencies of hazards for right- and left-sided tumors. Therefore, cigarette smoking might be instrumental in the mechanism for heterogeneity of sidedness.

\section{Abbreviations}

BACC: Bigdata Alliance for Colorectal Cancer; Cl: Confidence interval; CRC : Colorectal cancer; HR: Hazard ratio; LSCC: Left-sided colon cancer; LSCRC : Left-sided colorectal cancer; mCRC: Metastatic colorectal cancer; OR: Odds ratios; OS: Overall survival; RC: Rectal cancer; RSCC: Right-sided colon cancer; VIF: Variance inflation factor. 


\section{Supplementary Information}

The online version contains supplementary material available at https://doi. org/10.1186/s12967-021-02815-4.

Additional file 1: Figure S1. Flowchart of the patient selection process.

Additional file 2: Table S1. Assessment of multicollinearity of the regression models for the association analyses. Table S2. Odds ratios for sidedness of colorectal cancer according to smoking history adjusted for age, sex, family history and geographic location of medical centers. Table S3. Clinical features of the study cohort stratified by tumor sidedness. Table S4. Demographic and clinical characteristics of the cohort M ( $n=2272)$. Table S5. Survival statistics of patients with metastatic colorectal cancer tumor regarding sidedness and history of smoking $(n=2$ 272). Table S6. Death risks of cohort M regarding cigarette smoking on a quantitation basis.

\section{Acknowledgements}

We sincerely thank the staff of YiduCloud Technology Ltd. for providing technical support for the BACC platform.

\section{Authors' contributions}

All authors contributed to the study conception. Study design was performed by LPY, ZXW, XCZ, YPL and YJ. Data preparation, collection, coordination and quality control were performed by LPY, YJ, RZ, NZ, AMW, WL, JWL and FL. Data analysis and interpretation were performed by LPY, ZXW, ZQW, HYL and FW. The first draft of the manuscript was written by LPY and ZXW. All authors commented on previous versions of the manuscript. All authors read and approved the final manuscript.

\section{Funding}

The authors received no specific funding for this work.

\section{Availability of data and materials}

The key raw data have been deposited into the Research Data Deposit (http:// www.researchdata.org.cn) with the Approval Number of RDDA2020001769. The data analyzed during the current study are available from the corresponding author on reasonable request.

\section{Declarations}

\section{Ethics approval and consent to participate}

The Institute Research Ethics Committee at the Sun Yat-sen University Cancer Center approved the study (ID: B2018-154-01). The study was performed in accordance with the Declaration of Helsinki.

\section{Consent for publication}

Not applicable.

\section{Competing interests}

The authors declare that they have no competing interests.

\section{Author details \\ 'State Key Laboratory of Oncology in South China, Collaborative Innova- tion Center for Cancer Medicine, Sun Yat-Sen University Cancer Center, 651 Dong Feng Road East, Guangzhou 510060, China. ${ }^{2}$ Department of Colorectal Surgery, Cancer Hospital of China Medical University, Liaoning Cancer Hospital \& Institute, Shenyang 110042, China. ${ }^{3}$ Precision Medicine Center of Oncology, The Affiliated Hospital of Qingdao University, No. 59 Haier Road, Qing- dao 266016, China. ${ }^{4}$ Department of Oncology, The First Affiliated Hospital of Dalian Medical University, Dalian 116011, China. ${ }^{5}$ Department of Medical Oncology, The First Hospital of China Medical University, Shenyang 110001, China. ${ }^{6}$ Yiducloud Technology Ltd, Beijing 100083, China.}

Received: 6 January 2021 Accepted: 2 April 2021

Published online: 15 April 2021

\section{References}

1. Brenner H, Kloor M, Pox CP. Colorectal cancer. Lancet (London, England). 2014;383(9927):1490-502

2. Siegel R, Ma J, Zou Z, Jemal A. Cancer statistics, 2014. CA: A Cancer Journal for Clinicians. 2014;64(1):9-29.

3. Gay LJ, Mitrou PN, Keen J, Bowman R, Naguib A, Cooke J, et al. Dietary, lifestyle and clinicopathological factors associated with APC mutations and promoter methylation in colorectal cancers from the EPIC-Norfolk study. J Pathol. 2012;228(3):405-15.

4. Goncalves MD, Lu C, Tutnauer J, Hartman TE, Hwang SK, Murphy CJ, Pauli C, Morris R, Taylor S, Bosch K, Yang S. High-fructose corn syrup enhances intestinal tumor growth in mice. Science. 2019;363(6433):1345-9.

5. Botteri E, lodice S, Bagnardi V, Raimondi S, Lowenfels AB, Maisonneuve P. Smoking and colorectal cancer a meta-analysis. JAMA-J Am Med Assoc. 2008;300(23):2765-78

6. Liang PS, Chen TY, Giovannucci E. Cigarette smoking and colorectal cancer incidence and mortality: Systematic review and meta-analysis. Int J Cancer. 2009;124(10):2406-15.

7. Inoue M, Tajima K, Hirose K, Hamajima N, Takezaki T, Hirai T, et al. Subsite-specific risk factors for colorectal cancer: a hospital-based case-control study in Japan. Cancer Causes control. 1995;6(1):14-22.

8. Zisman AL, Nickolov A, Brand RE, Gorchow A, Roy HK. Associations between the age at diagnosis and location of colorectal cancer and the use of alcohol and tobacco: implications for screening. Arch Intern Med. 2006;166(6):629-34.

9. Sharpe CR, Siemiatycki JA, Rachet BP. The effects of smoking on the risk of colorectal cancer. Dis Colon Rectum. 2002;45(8):1041-50.

10. Hu J, Morrison H, Mery L, DesMeules M, Macleod M. Diet and vitamin or mineral supplementation and risk of colon cancer by subsite in Canada. European J Cancer Prevention. 2007;16(4):275-91.

11. Ben-Aharon I, Goshen-Lago T, Sternschuss M, Morgenstern S, Geva R, Beny A, et al. Sidedness Matters: Surrogate Biomarkers Prognosticate Colorectal Cancer upon Anatomic Location. The oncologist. 2019.

12. Petrelli F, Tomasello G, Borgonovo K, Ghidini M, Turati L, Dallera P, et al. Prognostic Survival Associated With Left-Sided vs Right-Sided Colon Cancer: A Systematic Review and Meta-analysis. JAMA oncology. 2016.

13. Tejpar S, Stintzing S, Ciardiello F, Tabernero J, Van Cutsem E, Beier F, et al. Prognostic and Predictive Relevance of Primary Tumor Location in Patients With RAS Wild-Type Metastatic Colorectal Cancer: Retrospective Analyses of the CRYSTAL and FIRE-3 Trials. JAMA oncology. 2016.

14. Jayasekara H, English DR, Haydon A, Hodge AM, Lynch BM, Rosty $C$, et al. Associations of alcohol intake, smoking, physical activity and obesity with survival following colorectal cancer diagnosis by stage, anatomic site and tumor molecular subtype. Int J Cancer. 2018;142(2):238-50.

15. Karim S, Brennan K, Nanji S, Berry SR, Booth CM. Association between prognosis and tumor laterality in early-stage colon cancer. JAMA Oncol. 2017;3(10):1386-92.

16. Altman DG, Bland JM. Statistics Notes-Interaction revisited: the difference between two estimates. BMJ British Medical J. 2003;326(7382):219.

17. Venook AP, Ou FS, Lenz HJ, Kabbarah O, Qu XP, Niedzwiecki D, et al. Primary (1 degrees) tumor location as an independent prognostic marker from molecular features for overall survival (OS) in patients (pts) with metastatic colorectal cancer (mCRC): Analysis of CALGB / SWOG 80405 (Alliance). J Clin Oncol. 2017;35:5.

18. Hecht SS. Tobacco carcinogens, their biomarkers and tobacco-induced cancer. Nat Rev Cancer. 2003;3(10):733-44.

19. Diergaarde B, Vrieling A, van Kraats AA, van Muijen GNP, Kok FJ, Kampman E. Cigarette smoking and genetic alterations in sporadic colon carcinomas. Carcinogenesis. 2003;24(3):565-71.

20. Carr PR, Alwers F, Bienert S, Weberpals J, Kloor M, Brenner H, et al. Lifestyle factors and risk of sporadic colorectal cancer by microsatellite instability status: a systematic review and meta-analyses. Ann Oncol. 2018;29(4):825-34.

21. Limsui D, Vierkant RA, Tillmans LS, Wang AH, Weisenberger DJ, Laird PW et al. Cigarette smoking and colorectal cancer risk by molecularly defined subtypes. J Natl Cancer Inst. 2010;102(14):1012-22.

22. Yamauchi M, Morikawa T, Kuchiba A, Imamura Y, Qian ZR, Nishihara R, et al. Assessment of colorectal cancer molecular features along bowe 
subsites challenges the conception of distinct dichotomy of proximal versus distal colorectum. Gut. 2012;61(6):847-54.

23. Natsume S, Yamaguchi T, Takao M, lijima T, Wakaume R, Takahashi K, et al. Clinicopathological and molecular differences between right-sided and left-sided colorectal cancer in Japanese patients. Jpn J Clin Oncol. 2018;48(7):609-18.

24. Shen $\mathrm{H}$, Yang J, Huang Q, Jiang MJ, Tan YN, Fu JF, et al. Different treatment strategies and molecular features between right-sided and left-sided colon cancers. World J Gastroenterol. 2015:21 (21):6470-8.

25. Loupakis F, Yang D, Yau L, Feng S, Cremolini C, Zhang W, et al. Primary tumor location as a prognostic factor in metastatic colorectal cancer. $J$ Natl Cancer Inst. 2015;107(3).

26. Kajizono M, Saito M, Maeda M, Yamaji K, Fujiwara S, Kawasaki Y, et al. Cetuximab-induced skin reactions are suppressed by cigarette smoking in patients with advanced colorectal cancer. Int J Clin Oncol. 2013;18(4):684-8.

27. Abdel-Rahman O, Cheung WY. Impact of smoking history on the outcomes of women with early-stage breast cancer: a secondary analysis of a randomized study. Med Oncol. 2018;35(5):68.

28. Hasegawa $Y$, Ando M, Maemondo M, Yamamoto S, Isa S, Saka H, et al. The role of smoking status on the progression-free survival of non-small cell lung cancer patients harboring activating epidermal growth factor receptor (EGFR) mutations receiving first-line EGFR tyrosine kinase inhibitor versus platinum doublet chemotherapy: a meta-analysis of prospective randomized trials. Oncologist. 2015;20(3):307-15.

29. Kawaguchi C, Sho M, Tanaka T, Akahori T, Kinoshita S, Nagai M, et al. Impact of smoking on pancreatic cancer patients receiving current chemotherapy. Pancreas. 2015;44(7):1155-60.

30. Kelemen LE, Warren GW, Koziak JM, Kobel M, Steed H. Smoking may modify the association between neoadjuvant chemotherapy and survival from ovarian cancer. Gynecol Oncol. 2016:140(1):124-30.

31. Desrichard A, Kuo F, Chowell D, Lee KW, Riaz N, Wong RJ, et al. Tobacco smoking-associated alterations in the immune microenvironment of squamous cell carcinomas. J Natl Cancer Inst. 2018;110(12):1386-92.

32. Zhou GB, Norum J, Nieder C. Tobacco smoking and cessation and PD-L1 inhibitors in non-small cell lung cancer (NSCLC): a review of the literature. Nat Commun. 2018;3(6):e000406.

\section{Publisher's Note}

Springer Nature remains neutral with regard to jurisdictional claims in published maps and institutional affiliations.
Ready to submit your research? Choose BMC and benefit from:

- fast, convenient online submission

- thorough peer review by experienced researchers in your field

- rapid publication on acceptance

- support for research data, including large and complex data types

- gold Open Access which fosters wider collaboration and increased citations

- maximum visibility for your research: over 100M website views per year

At BMC, research is always in progress.

Learn more biomedcentral.com/submissions 